\title{
Predictive impact of elevated serum level of IL-18 for early renal dysfunction in type 2 diabetes: an observational follow-up study
}

\author{
S. Araki • M. Haneda • D. Koya • T. Sugimoto • \\ K. Isshiki • M. Chin-Kanasaki • T. Uzu • A. Kashiwagi
}

Received: 31 July 2006 / Accepted: 4 December 2006 / Published online: 16 January 2007

(C) Springer-Verlag 2007

\begin{abstract}
Aims/hypothesis The early identification of type 2 diabetic patients at risk of developing microalbuminuria - an independent risk factor for renal and cardiovascular diseasesis important to improve the patients' outcomes. We investigated whether serum levels of IL-18, a proinflammatory cytokine, were a predictor of early renal dysfunction. Materials and methods A total of 249 Japanese type 2 diabetic patients without overt proteinuria were enrolled in an observational follow-up study (median follow-up 7 years), and their stage of diabetic nephropathy was classified and their estimated glomerular filtration rate (eGFR) was calculated annually.

Results At baseline, serum levels of IL-18 were higher in subjects with microalbuminuria $(n=76)$ than in those with normoalbuminuria $(n=173)$. Elevated serum levels of IL-18 were associated with the progression of nephropathy to a higher stage in normoalbuminuric subjects (118 [interquartile range 91-159] ng/l vs 155 [interquartile range 121-205] $\mathrm{ng} / \mathrm{l}, p=0.003$ ), but not in microalbuminuric subjects (154 [interquartile range 113-200] ng/l vs 160 [interquartile range
\end{abstract}

S. Araki $(\bowtie) \cdot$ T. Sugimoto $\cdot$ K. Isshiki $\cdot$ M. Chin-Kanasaki $\cdot$

T. Uzu $\cdot$ A. Kashiwagi

Department of Medicine, Shiga University of Medical Science,

Otsu, Shiga 520-2192 Japan

e-mail: araki@belle.shiga-med.ac.jp

M. Haneda

Second Department of Medicine,

Asahikawa Medical College,

Asahikawa, Japan

D. Koya

Division of Endocrinology and Metabolism,

Department of Medicine, Kanazawa Medical University,

Kanazawa, Japan
101-190] ng/l, $p=0.50)$. The adjusted risk for developing microalbuminuria was 3.6 (95\% CI 1.2-10.4) in normoalbuminuric subjects with serum IL-18 levels above the median $(\geq 134.6 \mathrm{ng} / \mathrm{l})$, and was significantly enhanced in those urinary AERs at the upper end of the normal range $(7.5 \mu \mathrm{g} / \mathrm{min} \leq$ AER $<20 \mu \mathrm{g} / \mathrm{min})$. Furthermore, the annual rate of decline in eGFR, when examined in the study population as a whole, was significantly greater in subjects with serum IL-18 levels above the median than in other subjects.

Conclusions/interpretation The results of our observational follow-up study indicate that elevated serum levels of IL-18 may be a predictor of future renal dysfunction in type 2 diabetic patients with normoalbuminuria.

Keywords Diabetic nephropathy · High-sensitivity C-reactive protein $\cdot$ IL-18 $\cdot$ Inflammation .

Microalbuminuria $\cdot$ Normoalbuminuria $\cdot$ Renal dysfunction

\section{Abbreviations \\ eGFR estimated glomerular filtration rate \\ hs-CRP high-sensitivity C-reactive protein \\ MDRD Modification of Diet in Renal Disease}

\section{Introduction}

Nephropathy associated with type 2 diabetes is a leading cause of end-stage renal disease. The earliest clinical sign of diabetic nephropathy is an elevated urinary AER, referred to as microalbuminuria [1]. Microalbuminuria in diabetic patients has been recognised not only as a predictor of progression of diabetic nephropathy, but also as a 
powerful independent risk factor for cardiovascular disease [2-5]. Epidemiological studies have demonstrated that only a limited proportion of diabetic patients develop microalbuminuria [1]; thus, early identification of at-risk patients and initiation of appropriate therapy is important for the improvement of outcomes.

Chronic subclinical inflammation has been proposed to be involved in the development of microalbuminuria in diabetes $[6,7]$. This hypothesis is supported by several cross-sectional studies reporting elevated levels of markers of inflammation, such as high-sensitivity C-reactive protein (hs-CRP), TNF- $\alpha$ and IL-1, in diabetic patients with diabetic nephropathy versus those without [8-10]. IL-18, previously termed IFN- $\gamma$-inducing factor, is a potent proinflammatory cytokine involved in both innate and acquired immune responses [11]. IL-18 initiates a cytokine cascade with concomitant expression of proinflammatory markers, and the overproduction of IL-18 has been reported in various human inflammatory and autoimmune diseases [12]. Thus, IL-18 may contribute to the development of diabetic nephropathy mediated by inflammation.

The aim of this study was to investigate whether the serum levels of IL-18 or hs-CRP could predict the development of early diabetic nephropathy in Japanese patients with type 2 diabetes, in a long-term observational follow-up study. In the present study, we assessed two outcomes: stage of diabetic nephropathy, and kidney function as indicated by the estimated glomerular filtration rate (eGFR).

\section{Subjects and methods}

Subjects and examination A total of 249 Japanese type 2 diabetic patients without overt proteinuria were recruited from individuals who regularly visited the outpatient clinic of the Department of Medicine at Shiga University of Medical Science during 1996-1998, as reported previously [13]. Briefly, in the initial 2 years (baseline period) patients clinically diagnosed as having type 2 diabetes according to the WHO criteria [14] provided multiple 24-h urine samples for assessment of urinary AER and eGFR. On the basis of multiple urinary AER measurements (mean 2.7, range 2-9 measurements), patients were classified (regardless of diabetes duration) as having normoalbuminuria, microalbuminuria or overt proteinuria. Only patients with normoalbuminuria or microalbuminuria at baseline were enrolled. Each individual provided a blood sample for biochemical measurements. Serum was stored at $-80^{\circ} \mathrm{C}$ if not analysed immediately. At each annual follow-up, participants underwent a standardised physical examination and provided a blood sample for biochemical measurements and a 24-h urine sample for determination of AER. Participants were followed up for at least 3 years, until the end of 2005 or death.

Serum levels of IL-18 were measured using a sandwich ELISA (MBL, Nagoya, Japan). In brief, diluted serum samples and standards were incubated on 96-well plates coated with anti-human IL-18 monoclonal antibody. After washing, a peroxidise-conjugated anti-human IL-18 monoclonal antibody was added to each microwell and incubated. After another washing, the peroxidase substrate was mixed with the chromogen and allowed to incubate for $30 \mathrm{~min}$ at room temperature. After terminating the enzyme reaction using an acid stop solution, the optical density of each well was measured at $450 \mathrm{~nm}$ using a microplate reader. The intraand inter-assay CVs for IL-18 were $5.6 \%$ and $6.3 \%$, respectively. The sensitivity of the assay was $12.5 \mathrm{ng} / \mathrm{l}$. Serum levels of hs-CRP were measured using a latex turbidimetric immunoassay, which had a sensitivity of $0.05 \mathrm{mg} / 1$ (Nanopia CRP; Daiichi Pure Chemicals, Tokyo, Japan). The intra- and inter-assay CVs for hs-CRP were $1.0 \%$ and $0.8 \%$, respectively. These parameters were measured twice for each individual at baseline, and the geometric mean was used as the baseline value in the analysis. Data for 249 patients (173 with normoalbuminuria, 76 with microalbuminuria at baseline) were used in the analysis.

The study protocol and informed consent procedure were approved by the Ethics Committee of Shiga University of Medical Science.

Definition of outcomes The stage of diabetic nephropathy was determined by the AER as measured by the immunoturbidimetry assay (Hitachi 7070E; Hitachi High-Technologies, Tokyo, Japan) in 24-h urine samples. Patients were designated as having normoalbuminuria (AER $<20 \mu \mathrm{g} / \mathrm{min}$ ), microalbuminuria $(20 \leq \mathrm{AER}[\mu \mathrm{g} / \mathrm{min}]<200)$ or overt proteinuria $\left(A E R \geq 200 \mu \mathrm{g} / \mathrm{min}\right.$ and $\mathrm{eGFR} \geq 60 \mathrm{ml} \mathrm{min}^{-1}$ $1.73 \mathrm{~m}^{-2}$; stage 1 or 2 according to the clinical practice guidelines set out by the Kidney Disease Outcomes Quality Initiative of the National Kidney Foundation [K/DOQI] [15]) based on at least two consecutive measurements. The outcome in terms of diabetic nephropathy stage was defined as the transition from any given stage to a more advanced stage of diabetic nephropathy for two consecutive years. Subjects who showed an intermittent transition to advanced stages were not classed as progressors.

The GFR was estimated using one of the equations developed in the Modification of Diet in Renal Disease (MDRD) study [16]. In the present study, data on daily urea excretion from 24-h urine collection was available for each 
individual. We therefore used the following MDRD equation, which includes demographic, serum and urine variables, and was reported to be the most precise in the original MDRD study [16]:

$$
\begin{aligned}
\mathrm{GFR}= & 198 \times[\mathrm{Pcr} \times 0.0113]^{-0.858} \times[\mathrm{age}]^{-0.167} \\
& \times[0.822 \text { if patient is female }] \\
& \times[\mathrm{SUN} \times 2.8011]^{-0.293} \times[\mathrm{UUN}]^{+0.249}
\end{aligned}
$$

where Pcr is serum creatinine concentration expressed as $\mu \mathrm{mol} / 1$ (original equation in [16] modified to incorporate SI units, with $\mathrm{mg} / \mathrm{dl}$ converted into $\mu \mathrm{mol} / \mathrm{l}$ ), SUN is serum urea nitrogen concentration expressed as $\mathrm{mmol} / \mathrm{l}$ (original equation in [16] modified to incorporate SI units, with $\mathrm{mg} / \mathrm{dl}$ converted into $\mathrm{mmol} / \mathrm{l}$ ), and UUN is urinary urea nitrogen excretion (g/day). The rate of annual decline in eGFR $(\triangle \mathrm{e} G F R)$ over the course of the study was determined from the slope of the plot of all measurements of eGFR for each individual (median eight measurements, range 4-14 measurements) calculated by linear regression analysis and was expressed as $\mathrm{ml} \mathrm{min}^{-1}$ $1.73 \mathrm{~m}^{-2}$ year $^{-1}$.

Statistical analysis Comparisons between subjects within each study group were made by $\chi^{2}$ tests with Fisher's exact test. Comparisons between groups were performed by using an unpaired Student's $t$ test for normally distributed variables and a Mann-Whitney $U$ test for non-normally distributed variables. Correlation was determined by a multivariate linear regression analysis with a stepwise backward method. Time to incidence of transition of nephropathy stage was estimated using the Kaplan-Meier method and statistical differences between groups were compared by the log-rank test. Follow-up time was censored if microalbuminuria developed or progressed, or if the patient was unavailable for follow-up. To adjust for the influence of conventional risk factors on the transition, a multivariate Cox proportional hazard regression model was applied. The independent variables used as conventional risk factors were sex, age, duration of diabetes, $\mathrm{HbA}_{1 \mathrm{c}}$, total cholesterol, triacylglycerol, AER, systolic BP, diastolic BP, hypertension (present/absent), the use of reninangiotensin system blockers (yes/no), retinopathy (present/ absent), BMI, current smoker and eGFR at baseline. Given that a target blood pressure of $<130 / 80 \mathrm{mmHg}$ is now recommended in diabetic patients [1], in this study, hypertension was defined as a $\mathrm{BP} \geq 130 / 80 \mathrm{mmHg}$ or current use of antihypertensive drugs. All data were analysed using the SPSS software package (version 11; SPSS, Chicago, IL, USA). A $p$ value of less than 0.05 was taken to be statistically significant.

\section{Results}

Table 1 shows the clinical characteristics of the participants according to stage of diabetic nephropathy at the baseline. At baseline, the proportion of men, duration of diabetes, $\mathrm{HbA}_{1 \mathrm{c}}$, triacylglycerol, systolic BP, diastolic BP, BMI, percentage of current smokers and AER were all significantly higher in the group of diabetic subjects with microalbuminuria than in the group with normoalbuminuria. Subjects with microalbuminuria had significantly lower levels of HDL-cholesterol compared with subjects with normoalbuminuria. Interestingly, serum levels of IL-18 were significantly elevated in subjects with microalbuminuria compared with those with normoalbuminuria, whereas serum hs-CRP levels were not different in the two groups. Significant independent correlations with serum levels of IL-18 were observed for sex (female: $\beta=-0.18, p=0.001$ ), $\log$ AER

\begin{tabular}{|c|c|c|}
\hline Clinical characteristic & $\begin{array}{l}\text { Normoalbuminuria } \\
(n=173)\end{array}$ & $\begin{array}{l}\text { Microalbuminuria } \\
(n=76)\end{array}$ \\
\hline Sex (male/female) & $78 / 95$ & $52 / 24^{*}$ \\
\hline Age (years) & $61 \pm 9$ & $61 \pm 8$ \\
\hline $\begin{array}{l}\text { Duration of diabetes } \\
\text { (years) }\end{array}$ & $13 \pm 8$ & $15 \pm 8^{*}$ \\
\hline $\mathrm{HbA}_{1 \mathrm{c}}(\%)$ & $7.1 \pm 0.8$ & $7.5 \pm 0.9^{*}$ \\
\hline $\begin{array}{l}\text { Diabetes treatment } \\
\text { (diet/oral agents/insulin) }\end{array}$ & $29 / 102 / 42$ & $8 / 45 / 23$ \\
\hline Total cholesterol (mmol/1) & $5.52 \pm 0.75$ & $5.35 \pm 0.75$ \\
\hline Triacylglycerol (mmol/l) & $1.06(0.75-1.46)$ & $1.16(0.96-1.65)^{*}$ \\
\hline HDL-cholesterol (mmol/l) & $1.47(1.24-1.81)$ & $1.34(0.91-1.58)^{*}$ \\
\hline BMI $\left(\mathrm{kg} / \mathrm{m}^{2}\right)$ & $23 \pm 3$ & $24 \pm 3^{*}$ \\
\hline Waist circumference $(\mathrm{cm})$ & $86 \pm 9$ & $88 \pm 9$ \\
\hline $\begin{array}{l}\text { Systolic blood pressure } \\
(\mathrm{mmHg})\end{array}$ & $134 \pm 15$ & $138 \pm 16^{*}$ \\
\hline $\begin{array}{l}\text { Diastolic blood pressure } \\
(\mathrm{mmHg})\end{array}$ & $76 \pm 8$ & $79 \pm 8^{*}$ \\
\hline Hypertension (\%) & $70 \%$ & $83 \%$ \\
\hline $\begin{array}{l}\text { Antihypertensive drugs } \\
\text { (none/ACE inhibitor or } \\
\text { ARB/others) }\end{array}$ & $108 / 29 / 36$ & $42 / 21 / 13$ \\
\hline Current smoker $(\%)$ & 18 & $37 *$ \\
\hline Retinopathy (\%) & 89 & 93 \\
\hline $\operatorname{AER}(\mu \mathrm{g} / \mathrm{min})$ & $7.4(5.7-11.1)$ & $48.7(35.3-74.5)^{*}$ \\
\hline $\begin{array}{l}\text { eGFR (min } \mathrm{ml}^{-1} \\
\left.1.73 \mathrm{~m}^{-2}\right)\end{array}$ & $105 \pm 19$ & $104 \pm 21$ \\
\hline IL-18 (ng/l) & $120(94-168)$ & $154(111-197)^{*}$ \\
\hline hs-CRP (mg/l) & $0.6(0.3-1.2)$ & $0.7(0.4-1.3)$ \\
\hline
\end{tabular}

Table 1 Clinical characteristics at baseline in subgroups according to the clinical stages of diabetic nephropathy

Data are expressed as means \pm SD for normally distributed continuous variables or medians (interquartile range) for skewed continuous variables

$* p<0.05$ vs normoalbuminuria

ARB: angiotensin II receptor blocker 
$(\beta=0.22, p<0.001), \log$ HDL-cholesterol $(\beta=-0.20$, $p<0.001)$ and $\log$ hs-CRP $(\beta=0.15, p=0.006)$ in a multivariate linear regression analysis with stepwise method.

We next explored the predictive effect of IL-18 and hsCRP on the development and progression of diabetic nephropathy. During the follow-up period (median 7 years, range $3-8$ years), 25 of the 173 diabetic subjects with normoalbuminuria and 21 of the 76 with microalbuminuria showed a transition from any given stage to a more advanced stage of diabetic nephropathy. Among subjects with normoalbuminuria at baseline, serum IL-18 levels were elevated in those whose nephropathy progressed to a more advanced stage compared with those who remained normoalbuminuric (118 [interquartile range $91-159$ ] ng/l vs 155 [interquartile range 121-205] ng/l, $p=0.003$ by MannWhitney $U$ test), whereas, among subjects with microalbuminuria at baseline, IL-18 levels were not different between these two subgroups (154 [interquartile range 113200] $\mathrm{ng} / \mathrm{l}$ vs 160 [interquartile range 101-190] $\mathrm{ng} / \mathrm{l}, p=$ 0.50 ). This trend remained even when the analysis was adjusted for sex. Serum levels of hs-CRP did not differ between the non-progressors and progressors in either the normoalbuminuria group (0.6 [interquartile range $0.3-1.2$ ] $\mathrm{mg} / \mathrm{l}$ vs 0.8 [interquartile range: $0.3-1.3$ ] $\mathrm{mg} / \mathrm{l}$ ) or the microalbuminuria group (0.7 [interquartile range: $0.4-1.4$ ] $\mathrm{mg} / \mathrm{l}$ vs 0.7 [interquartile range: $0.3-1.2$ ] $\mathrm{mg} / \mathrm{l}$ ). Furthermore, serum levels of IL-18 ( $\geq 134.6 \mathrm{ng} / \mathrm{l})$ above the median were predictive of the progression of nephropathy to a higher stage in patients with normoalbuminuria, as examined with the Kaplan-Meier method (log-rank test $p=$ 0.005), but not in those with microalbuminuria (Fig. 1). The adjusted risk for subjects with normoalbuminuria and serum IL-18 levels above the median level was 3.6 (95\% CI 1.210.4) after adjustment for conventional risk factors in the multiple hazard Cox model. In addition, neither serum levels of IL-18 nor hs-CRP were associated with mortality, although only six patients died during the follow-up period.
To further evaluate the predictive value of serum IL-18 in subjects with normoalbuminuria, these subjects were categorised as being above (high normal: $7.5 \mu \mathrm{g} / \mathrm{min}$ $\leq$ AER $<20 \mu \mathrm{g} / \mathrm{min}$ ) or below (low normal: AER $<7.5$ $\mu \mathrm{g} / \mathrm{min})$ the median value for urinary AER. Normoalbuminuric subjects with serum IL-18 levels above the median and a high normal AER had an increased risk of developing microalbuminuria (Fig. 2). Their adjusted risk compared with those with serum IL-18 levels below the median and a low normal AER was 12.9 (95\% CI 2.9-58.4) in the multiple hazard Cox model after adjustment for conventional risk factors, whereas the risk was 1.7 (95\% CI 0.2 12.3 ) in subjects with above-median serum IL-18 levels and a low normal AER, and 2.7 (95\% CI 0.5-15.2) in those with below-median serum IL-18 levels and a high normal AER.

Finally, we investigated the effect of serum IL-18 on kidney function as evaluated by the annual rate of change in eGFR in the entire study population. In subjects with above-median serum IL-18, the rate of annual change in eGFR was significantly higher than in those with belowmedian serum IL-18 ( -2.1 [interquartile range -0.5 to -3.0 ] $\mathrm{ml} \mathrm{min}{ }^{-1} 1.73 \mathrm{~m}^{-2}$ year $^{-1}$ vs -1.1 [interquartile range -0.2 to -2.9$] \mathrm{ml} \mathrm{min}^{-1} 1.73 \mathrm{~m}^{-2}$ year $^{-1}, p=0.036$ ) (Fig. 3).

\section{Discussion}

The present study provides evidence of the prognostic impact of elevated serum IL-18 on future development of microalbuminuria in normoalbuminuric patients with type 2 diabetes mellitus. The effect of serum IL-18 was significantly enhanced in patients with high normal levels of urinary AER. Furthermore, elevated serum levels of IL-18 were associated with an accelerated annual rate of decline in eGFR. Thus, the measurement of serum IL-18 may be very useful for the identification of subjects at high risk of
Fig. 1 Kaplan-Meier curves for the development and progression of microalbuminuria according to serum levels of IL18. a Subjects with normoalbuminuria at baseline ( $p=0.005$ by log-rank test). b Subjects with microalbuminuria at baseline ( $p=0.83$ by log-rank test). Subjects with IL-18 $\geq 134.6 \mathrm{ng} / 1(\longrightarrow)$; subjects with IL-18<134.6 ng/1(------)
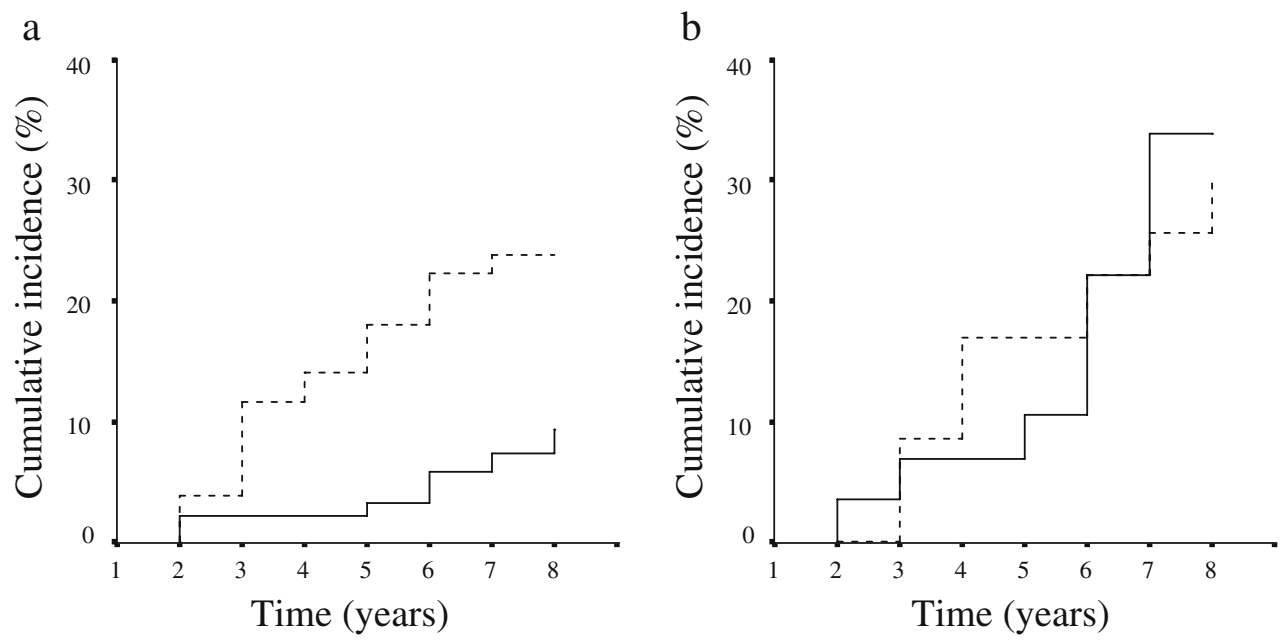


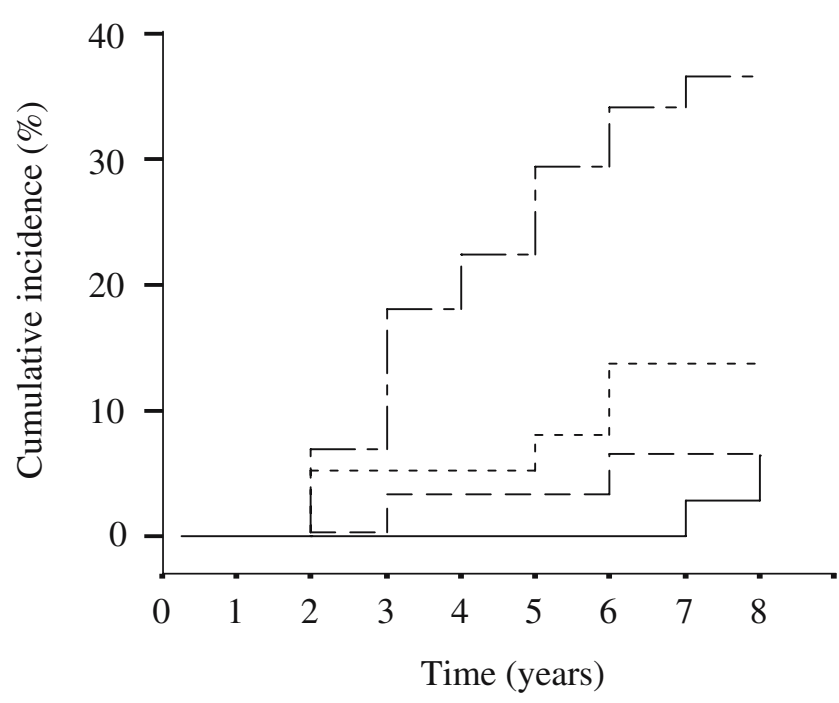

Fig. 2 Kaplan-Meier curves for the development of microalbuminuria in subjects with normoalbuminuria. The subjects were categorised as being above or below the median for serum IL-18 levels (134.6 ng/l) and urinary AER $(7.5 \mu \mathrm{g} / \mathrm{min}) .(\longrightarrow)$, low IL-18 + low AER $(n=$ 54); (- - $)$, high IL-18 + low AER $(n=33) ;(-----)$, low IL-18 + high AER $(n=41) ;(---)$, high IL-8 + high AER $(n=45) . p$ value for trend was $<0.001$

future renal dysfunction, which is an independent risk factor for renal and cardiovascular diseases.

The present findings for IL-18 examined in a long-term follow-up study extend the findings of previous crosssectional association studies in patients with type 1 and type 2 diabetes mellitus [17-19]. Nakamura et al. reported that serum and urinary IL-18 levels in Japanese patients with type 2 diabetes mellitus correlated positively with urinary AER at baseline and after 6 months, a relatively short period [19]. Our longer observational follow-up study clearly showed a prognostic impact of serum IL-18 levels on the future development of microalbuminuria. Thus, these results strongly suggest that the upregulation of IL-18 contributes to the development of diabetic kidney disease in type 2 diabetic patients.

Epidemiological studies have shown that only a limited number of patients with type 2 diabetes develop diabetic nephropathy [1]. High levels of urinary AER, even within the range of normoalbuminuria, are reported to be predictive of progression to microalbuminuria or proteinuria in patients with type 2 diabetes mellitus [20,21]. The present study clearly showed that high serum IL-18 levels are a strong predictor of the development of microalbuminuria in patients with normal but relatively high levels of urinary AER. This combination of serum IL-18 and urinary AER might come to be clinically considered as a useful way of identifying patients at risk of developing microalbuminuria.

The prognostic impact of serum IL-18 was not observed in patients with microalbuminuria. A plausible explanation is that microalbuminuria per se may be a consequence and/ or the cause of a chronic state of subclinical inflammation.
There is growing evidence that in diabetic patients, as well as in non-diabetic individuals, microalbuminuria is associated with endothelial dysfunction and subclinical inflammation [22]. Microalbuminuria may reflect diffuse endothelial damage secondary to a chronic state of subclinical inflammation. Elevated serum IL-18 may be a reflection of this state, which subsequently leads to the development of microalbuminuria. In fact, the levels of serum IL-18 in normoalbuminuric patients who later developed microalbuminuria were similar to those in patients with microalbuminuria at baseline. Nevertheless, the diabetic patients with lower levels of serum IL-18 may have some genetic protection against inflammation. The inhibitory molecules involved in the IL-18 inflammatory cascade may serve as a protective mechanism in such patients. A further study is required to elucidate this point.

Microalbuminuria has been shown to be associated with increased cardiovascular mortality in both diabetic and nondiabetic study populations [2-5]. The relative contributions of diabetic nephropathy to microalbuminuria and underlying vascular disease are not clear. Inflammation and activated innate immunity are considered to play an important role, not only in the development of microalbuminuria, but also in atherosclerosis and cardiovascular diseases. Although the regulation of IL-18 is still poorly understood, IL-18 is now recognised as a central regulator of innate and acquired immune responses and as a pleiotropic proinflammatory cytokine, playing an early role in the inflammatory cascade [12]. Thus, IL-18 may play a pivotal role in the pathophysiological mechanisms underlying the relationship between microalbuminuria and cardiovascular disease.

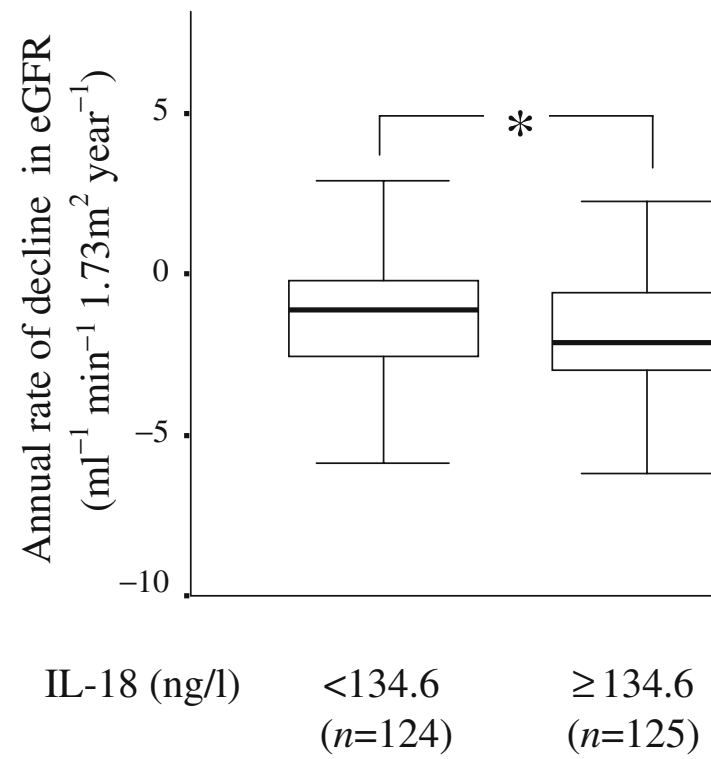

Fig. 3 The annual rate of decline in eGFR for patients grouped according to serum levels of IL-18. Horizontal bars represent medians, columns indicate interquartile ranges, and vertical bars represent $95 \%$ CIs. ${ }^{*} p=0.036$ by Mann-Whitney $U$ test 
In the present study, serum hs-CRP levels were not associated with the progression of diabetic nephropathy. Previous cross-sectional studies have observed high serum levels of hs-CRP in patients with diabetic nephropathy, suggesting a link between subclinical (low-grade) inflammation and diabetic nephropathy [8]. However, in our study serum levels of hs-CRP were not associated with the stage of diabetic nephropathy or the development of microalbuminuria. Although the reason for these discrepancies remains unclear, we suspect that IL-18 may more sensitively reflect chronic subclinical inflammation than hs-CRP, or may specifically reflect local inflammation in the kidney rather than systemic subclinical inflammation.

Several study limitations are acknowledged. In the present study, we did not evaluate the time-dependent changes of serum IL-18 levels during the follow-up period. Serum levels of IL-18 may be modified by certain medications, such as the renin-angiotensin blockers, which are reported to inhibit the production of IL-18 [23]. At baseline (1996-1998), approximately $20-30 \%$ of participants in the present study had taken an ACE inhibitor or an angiotensin II receptor blocker, whereas over $70 \%$ of diabetic patients at our hospital are now prescribed these medications (S. Araki, unpublished data). Thus, a further study is required to answer the interesting question of whether the decrease in serum IL-18 levels induced by medication can modify the outcome. Also, the pathophysiological mechanisms by which the high serum levels of IL18 predict the development of microalbuminuria remain to be elucidated. Furthermore, the source of the increased serum IL-18 in diabetic patients is not known. The upregulation of IL-18 in the diabetic kidney or infiltrated macrophage in the kidney may be responsible for the increased levels. However, this phenomenon may merely reflect the systemic chronic inflammation and not be specific to the kidney. Further human and animal studies are needed to elucidate these points.

In conclusion, our long-term observational follow-up study shows that a combination of high serum levels of IL18 and high but normal AER has a strong prognostic impact on the future development of microalbuminuria in diabetic patients with normoalbuminuria. This effect is independent of known conventional risk factors. Elevated levels of serum IL-18 were also associated with an accelerated decline in renal function. Therefore, measuring serum IL-18 in addition to urinary AER may allow the early identification of diabetic patients at risk of renal so that the appropriate medication may be initiated for the preservation of these complications.

Acknowledgements This work was partly supported by a Grant-inAid for Scientific Research from the Ministry of Health, Labor and Welfare of Japan. We would like to acknowledge S. Matuki (Daiichi
Fine Chemical Company) for technical support regarding the measurements of serum IL-18 and hs-CRP, and M. Yamanaka for data management.

Duality of interest None of the authors have any relevant dualities of interest to declare.

\section{References}

1. American Diabetes Association (2004) Nephropathy in diabetes. Diabetes Care 27:S79-S83

2. Dinneen SF, Gerstein HC (1997) The association of microalbuminuria and mortality in non-insulin-dependent diabetes mellitus. A systematic overview of the literature. Arch Intern Med 157:1413-1418

3. Garg JP, Bakris GL (2002) Microalbuminuria: marker of vascular dysfunction, risk factor for cardiovascular disease. Vasc Med 7:35-43

4. Adler AI, Stevens RJ, Manley SE, Bilous RW, Cull CA, Holman RR, UKPDS Group (2003) Development and progression of nephropathy in type 2 diabetes: the United Kingdom Prospective Diabetes Study (UKPDS 64). Kidney Int 63:225-232

5. Lane JT (2004) Microalbuminuria as a marker of cardiovascular and renal risk in type 2 diabetes mellitus: a temporal perspective. Am J Physiol Renal Physiol 286:F442-F450

6. Stuveling EM, Bakker SJ, Hillege HL, de Jong PE, Gans RO, de Zeeuw D (2005) Biochemical risk markers: a novel area for better prediction of renal risk? Nephrol Dial Transplant 20:497-508

7. Navarro JF, Mora C (2005) Role of inflammation in diabetic complications. Nephrol Dial Transplant 20:2601-2604

8. Navarro JF, Mora C, Maca M, Garca J (2003) Inflammatory parameters are independently associated with urinary albumin in type 2 diabetes mellitus. Am J Kidney Dis 42:53-61

9. Stehouwer CD, Gall MA, Twisk JW, Knudsen E, Emeis JJ, Parving HH (2002) Increased urinary albumin excretion, endothelial dysfunction, and chronic low-grade inflammation in type 2 diabetes: progressive, interrelated, and independently associated with risk of death. Diabetes 51:1157-1165

10. Lin J, Hu FB, Rimm EB, Rifai N, Curhan GC (2006) The association of serum lipids and inflammatory biomarkers with renal function in men with type II diabetes mellitus. Kidney Int 69:336-342

11. Okamura H, Tsutsui H, Komatsu T et al (1995) Cloning of a new cytokine that induces IFN-gamma production by T cells. Nature 378:88-91

12. Mühl H, Pfeilschifter J (2004) Interleukin-18 bioactivity: a novel target for immunopharmacological anti-inflammatory intervention. Eur J Pharmacol 500:63-71

13. Araki S, Haneda M, Sugimoto T et al (2006) Polymorphisms of the protein kinase $\mathrm{C}-\beta$ gene (PRKCB1) accelerate kidney disease in type 2 diabetes without overt proteinuria. Diabetes Care 29:864-868

14. WHO Study Group (1985) Diabetes mellitus. Technical report Series No. 727. WHO, Geneva

15. National Kidney Foundation (2002) K/DOQI clinical practice guidelines for chronic kidney disease: evaluation, classification, and stratification. Am J Kidney Dis 39(2 Suppl 1):S1-S266

16. Levey AS, Bosch JP, Lewis JB, Greene T, Rogers N, Roth D, Modification of Diet in Renal Disease Study Group (1999) A more accurate method to estimate glomerular filtration rate from serum creatinine: a new prediction equation. Ann Intern Med $130: 461-470$ 
17. Moriwaki Y, Yamamoto T, Shibutani Y et al (2003) Elevated levels of interleukin-18 and tumor necrosis factor-alpha in serum of patients with type 2 diabetes mellitus: relationship with diabetic nephropathy. Metabolism 52:605-608

18. Mahmoud RA, el-Ezz SA, Hegazy AS (2004) Increased serum levels of interleukin-18 in patients with diabetic nephropathy. Ital J Biochem 53:73-81

19. Nakamura A, Shikata K, Hiramatsu M et al (2005) Serum interleukin-18 levels are associated with nephropathy and atherosclerosis in Japanese patients with type 2 diabetes. Diabetes Care 28:2890-2895

20. Gall MA, Hougaard P, Borch-Johnsen K, Parving HH (1997) Risk factors for development of incipient and overt diabetic nephrop- athy in patients with non-insulin dependent diabetes mellitus: prospective, observational study. BMJ 314:783-788

21. Retnakaran R, Cull CA, Thorne KI, Adler AI, Holman RR (2006) Risk factors for renal dysfunction in type 2 diabetes: UK Prospective Diabetes Study 74. Diabetes 55:18321839

22. Saraheimo M, Teppo AM, Forsblom C, Fagerudd J, Groop PH (2003) Diabetic nephropathy is associated with low-grade inflammation in type 1 diabetic patients. Diabetologia 46:14021407

23. Lapteva N, Ide K, Nieda M et al (2002) Activation and suppression of renin-angiotensin system in human dendritic cells. Biochem Biophys Res Commun 296:194-200 